\title{
Roll Angle Determination of Fast Rotating Object and Orientation System Construction
}

\author{
A. Timoshenkov \\ Microelectronics Department \\ National Research University of Electronic Technology (MIET) \\ Zelenograd, Moscow, Russian Federation \\ porcuapa@gmail.com
}

\begin{abstract}
The problem of determining the roll angle of a fast rotating object has been analyzed in the paper. Possibility of creating a fast rotating single-axis orientation system that was able to determine the roll angle for a long time with minimal errors, based on data from various sources, has been considered. Algorithms designed to determine the roll angle of a fast rotating object using information from a micromechanical gyroscope, vector magnetometer and optical sensor have been considered in detail. Kalman filter which estimates the model of angular velocity sensor errors and the orientation error was used
\end{abstract}

Keywords-roll angle, sensor, MEMS gyro, signal, magnetometer, error.

\section{I.INTRODUCTION}

Today microsystem technologies are widely developed providing creation of various nano- and microelectromechanical systems (MEMS). Use of microelectronic technology adapted for the production of complex integrated devices that combine mechanical and electronic components in a single system is the main aspect in this direction [1].

MEMS consumers are many industries, including exploration, automotive, instrument engineering, aviation, transport, mining, medical equipment, sports simulators, telecommunications equipment, robotics, household appliances, defense industries, etc. Inertial sensors, including the angular velocity sensors (gyroscopes) have taken an important place in the MEMS series [2].

An advantage of MEMS inertial measurement units was based on mass production in group technology. This is the low price of the final product, reliability and repeatability of characteristics, miniature devices, low power consumption, shock and vibration resistance, which significantly expands the scope of such products. MEMS in general and angular velocity sensors in particular have a great potential for development, so it was used in various areas. An important attribute of the development of inertial MEMS is new functional capabilities of systems using microsystem technology. [1-3] Indeed, thanks to their small size, impact resistance and other qualities, sometimes it becomes possible to apply sensors in completely new areas with a completely new quality.

This work was supported by the Russian Science Foundation. Unique identifier for applied research is 16-19-00177
Thus, using of a very miniature gyroscope together with accelerometer and a number of other sensors, it becomes possible to create a whole orientation module in miniature objects in small diameter and volume. High resistance of sensors to aggressive external influences allows measuring the dynamics, parameters of movement and orientation of elements of drilling rigs in geological prospecting, sports game shells, aircrafts UAVs and other objects. The most important quality of such systems is the size of the sensors, and, concerning fast rotating object - the minimum radius of the arrangement of the sensor components. With the minimum mass and location radius, the most dynamic characteristics (limiting acceleration of angular velocity and acceleration) become available for objective evaluation. A large mass can distort the dynamics of the researched object. The aim of this research was to find the ways of the most accurate determination of the roll angle of a fast rotating object.

It is possible to design angular velocity converters with specified characteristics (range of measured angular velocities, bandwidth, and digital or analog output signal) for a wide range of consumers using modern computer-aided design (CAD) systems. The existing metrological base allows estimating the necessary characteristics of the manufactured GYRO for setting their compliance with technical requirements. However, there are many unresolved problems. One of the urgent problems is the justification and choice of the sensitive element (SE) geometry and principle of its operation, providing the possibility of angular velocity converters with the required sensitivity and resistance to external influencing factors.

Testing process to evaluate the parameters of produced samples is an integral process part of manufacturing products. Development of test methods and stands with hardware, software and algorithmic support is also important and necessary task.

Providing the possibility of GYROs creating with different ranges of angular velocities, which resistant to external influencing factors, required metrological and operational characteristics. Such studies are modern and relevant.

Motivation of this research was consisted in the development of angular velocity converters, methods for their adjustment, measurement of characteristics, and search for new functional applications of these devices. 
In this paper, possibility of creating a fast rotating singleaxis orientation system capable of determining the roll angle with minimal errors for a long time, based on data from various sources, such as a magnetometer and an optical sensor has been considered. Kalman filter will be used, which will be able to estimate model error for the angular velocity sensor and orientation error.

\section{II.EXPERIMENTAL PART AND RESULTS}

\section{Linear model of angular velocity sensor errors}

An angular velocity sensor (AVS or gyroscope) with the axis of sensitivity coinciding with the main axis of the fastrotating carrier serves as the main source of information about angular orientation of the carrier along the roll. Such errors of micromechanical AVS as a zero shift and a scale factor error lead to a rapid error accumulation in determining the angle, which makes direct application of AVS impossible. For evaluation and compensation of errors, it is proposed to apply the information integration obtained from AVS, from the vector magnetometer, as well as from the optical sensor. Modulation frequency of the signals, the magnetometer and optical sensor must generally coincide with the object's rotation frequency. The integration scheme was based on the Kalman filter application, which evaluates the AVS error model and the orientation error:

$\delta \dot{\alpha}=\Omega_{\mathrm{x}} \delta \mathrm{B}_{\mathrm{x}}+\delta \Omega_{0, \mathrm{x}}+v$

where $\delta \dot{\alpha}$ - error in determining the orientation angle around the $\mathrm{x}$ axis in the starting coordinate system, $\delta \mathrm{B}_{\mathrm{x}}$ - error in the scale factor of the AVS, $\delta \Omega_{0, \mathrm{x}}$ - zero shift of the AVS, $v$ - Gaussian noise with intensity q, and $\Omega$ - angular velocity of the carrier rotation about the $\mathrm{x}$ axis.

\section{System equations}

The state vector for the Kalman filter is written as:

$$
\mathrm{x}=\left[\begin{array}{lll}
\delta \alpha & \delta \mathrm{B}_{\mathrm{x}} & \delta \Omega_{0, \mathrm{x}}
\end{array}\right]^{\mathrm{T}}
$$

Then the system equations in a continuous form can be written as:

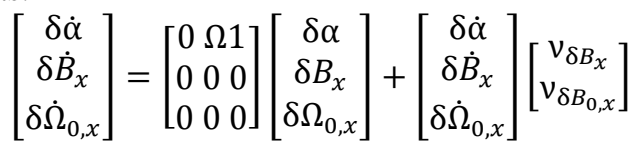

In abbreviated form:

$$
\dot{x}=\mathrm{Ax}+\mathrm{B} v
$$

Use the well-known relations for the transition to the discrete form of system equations:

$$
\boldsymbol{x}(k+1)=F(k) \boldsymbol{x}(k)+\boldsymbol{v}(k)
$$

$$
\begin{aligned}
& F=e^{A \Delta t} \\
& \boldsymbol{v}(k)=\int_{0}^{\Delta t} e^{A(\Delta t-\tau)} B \boldsymbol{v}(k \Delta t+\tau) d \tau \\
& \mathrm{Q}(\mathrm{k})=\mathrm{E} v(\mathrm{k}) v(\mathrm{k}) \mathrm{T}
\end{aligned}
$$

Then let us write the discrete matrix of system $\mathrm{F}(\mathrm{k})$ and noise matrix $\mathrm{Q}(\mathrm{k})$ as follows:

$$
\begin{gathered}
F(k)=\left[\begin{array}{ccc}
1 & \Omega \Delta t & \Delta t \\
0 & 1 & 0 \\
0 & 0 & 1
\end{array}\right] \\
F(k)=\left[\begin{array}{ccc}
\frac{v_{\delta B_{x}} \Delta t^{3} \Omega^{2}}{3}+\frac{v_{\delta \Omega_{0, x}} \Delta t^{3}}{3} & \frac{\Delta t^{2} v_{\delta B_{x} \Omega}}{2} & \frac{\Delta t^{2} v_{\delta B \Omega_{0, x}}}{2} \\
\frac{\Delta t^{2} v_{\delta B_{x} \Omega}}{2} & v_{\delta B_{0, x}} \Delta t & 0 \\
\frac{\Delta t^{2} v_{\delta B \Omega_{0, x}}}{2} & 0 & v_{\delta B_{0, x}} \Delta t
\end{array}\right],
\end{gathered}
$$

where $\Delta \mathrm{t}$ is the discretization period

\section{Equations of Measurements}

\section{Magnetometer measurements}

The rotation angle of the carrier around the $x$ axis can be measured with a magnetic field sensor:

$$
\alpha_{\mathrm{m}}=\operatorname{tg}^{-1} \frac{\mathrm{m}_{\mathrm{z}}}{\mathrm{m}_{\mathrm{y}}}+\eta
$$

where $m_{z}$ and $m_{y}$ are the components of the magnetic field measured by a calibrated magnetometer, $\eta$ is the Gaussian noise.

It should be noted that it is necessary to take into account the fact that angle $\alpha_{\mathrm{m}}$ is bounded by range $[-\pi, \pi]$, when calculating the difference. Measurement vector $\mathrm{z}$ can be written as follows:

$$
\mathrm{z}=\left[\begin{array}{lll}
\alpha_{\Omega}-\alpha_{\mathrm{m}} & 0 & 0
\end{array}\right]^{T}
$$

where $\alpha_{\Omega}$ is the obtained angle by integrating the angular velocity. Then, measurement matrix $\mathrm{H}$ is:

$$
H=\left[\begin{array}{lll}
1 & 0 & 0
\end{array}\right]
$$

\section{Optical sensor measurements}

Let us consider the ideal case in which an optical sensor, observing one light source, forms a sinusoidal signal of some amplitude with some average value at its output. In this case, the following AVS correction strategy is proposed:

- At the initial stage, the roll angle is calculated from the indications of only one AVS

- A signal from the light sensor is differentiated and, as soon as the algorithm detects zero derivative, the current AVS reading is taken as the standard value of the roll angle. 
Each subsequent revolution of the accumulated roll angle is compared with the reference value of the roll angle. The corresponding element of the measurement vector is formed and the correction of the roll sensor readings is performed in the Kalman filtering procedure.

The accumulated AVS roll angle is compared with the standard roll angle each subsequent turn. Due to this, the corresponding element of the measurement vector is formed and correction of the roll sensor readings is performed in the Kalman filter procedure.

It can be expected that in case of such approach, the error in the roll angle should not exceed the error accumulated by the AVS at a sufficiently short initial stage. Its current accuracy is determined by the error in determining the transition of the derivative signal through zero. The vector of measurements is similar to the case of magnetometer measurements and is written as follows:

$$
\mathrm{z}=\left[\begin{array}{lll}
\alpha_{\Omega}-\alpha_{\text {ref }} & 0 & 0
\end{array}\right]^{T}
$$

where $\alpha_{r e f}$ - standard angle.

\section{Kalman filter}

In the indirect Kalman filter, the error estimates obtained in the previous step are taken into account at the current step of the system. [4] It is most convenient to use the discrete filter algorithm in the Joseph form and write the equations as follows:

$$
\mathrm{P}=\mathrm{FP} \mathrm{F}^{\mathrm{T}}+\mathrm{Q}
$$

$$
\mathrm{K}=\left(\mathrm{P} \mathrm{H}^{\mathrm{T}}\right)\left(\mathrm{HPH}^{\mathrm{T}}+\mathrm{R}\right)^{-1}
$$

$\mathrm{P}=(\mathrm{I}-\mathrm{KH}) \mathrm{P}(\mathrm{I}-\mathrm{KH})^{\mathrm{T}}+\mathrm{KRK}^{\mathrm{T}}$

$\hat{x}=\mathrm{K}_{\mathrm{z}}$

Vector $\hat{x}$ contains the error estimate for the angle and estimation of the AVS error increments:

$$
\hat{x}=\left[\begin{array}{lll}
\delta \hat{\alpha} & \delta \widehat{\Omega_{0, x}}(k) & \delta \widehat{\mathrm{B}_{x}(k)}
\end{array}\right]^{T}
$$

Calculated estimation of AVS error increments is used to correct the current error values:

$$
\delta \widehat{\Omega}_{0, \mathrm{x}}(k)=\delta \widehat{\Omega}_{0, \mathrm{x}}(k-1)+\Delta \Omega_{0, x}(k)
$$

$\delta \widehat{\mathrm{B}}_{\mathrm{x}}(k)={ }_{\mathrm{x}}(k-1)+\Delta \mathrm{B} x(k)$

Angular velocity estimate $\widehat{\Omega}$, from which the orientation angle of the gyro $\alpha_{\Omega}$ is calculated, is as follows:

$$
\widehat{\Omega}(k)=\left(1-\delta \widehat{B}_{\mathrm{x}}(k)\right) \Omega(k)-\delta \widehat{\Omega}_{0, x}(k)
$$

A corrected roll angle is:

$$
\hat{\alpha}(k)=\alpha_{\Omega}(k)-\delta \hat{\alpha}
$$

\section{Modeling operation of the algorithm correction}

The model was developed in the Simulink for trajectory conditions construction (Figure 1).
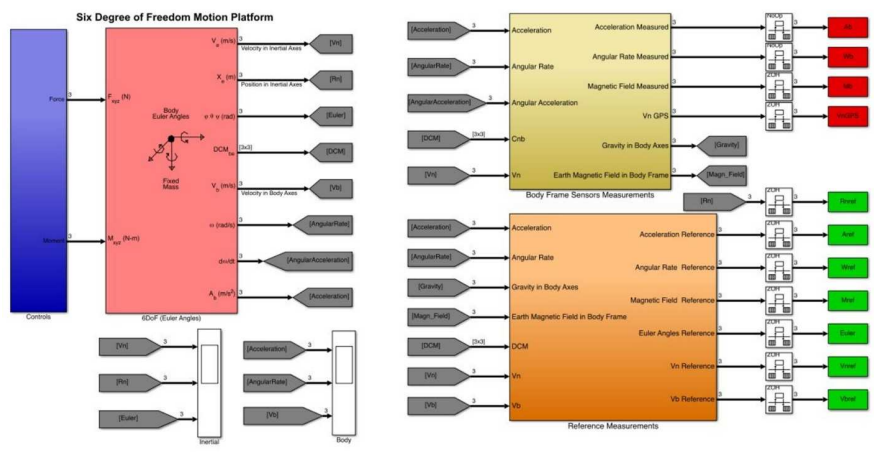

Fig. 1. Simulink-diagram model of the trajectory conditions builder

The basis is a dynamic model of a solid body moving under the action of the forces and moments applied to it. Standard kinematic information including angular velocities, linear accelerations in the associated coordinate system, as well as the velocities and coordinates of the center body mass in the navigation coordinate system serve for the construction of inertial sensors' (accelerometers and gyroscopes) indications, as well as a vector magnetometer [9].

The Earth's magnetic field model is used to build the magnetometer readings. Accelerometer readings are constructed taking into account the WGS84 model of the Earth's gravitational field.

Virtual measurements were formed based on the standard kinematic parameters, taking into account the set of sensor error models. These measurements were subjected to discretization with a given frequency. This information was used as an input to the algorithm. Presence of standard signals allowed us to evaluate the quality of its operation.

\section{Modeling algorithm with magnetometer correction}

The torque of the variable quantity around the $x$ axis was applied on the solid body model to simulate the operation of the roll angle correction algorithm [5,7,8]. Figure 2 shows the angular velocity and magnetic field components in the simulation process. The following model of AVS errors was adopted:

- AVSzero shift $\delta \Omega_{0, x}=0.01 \mathrm{rad} / \mathrm{s}$

- Scale factor error of the AVS $\delta \hat{B}_{x}=-0.05$

- Noise level of the $\mathrm{AVS} \sigma_{2}=0.001 \mathrm{rad} / \mathrm{s}$ 

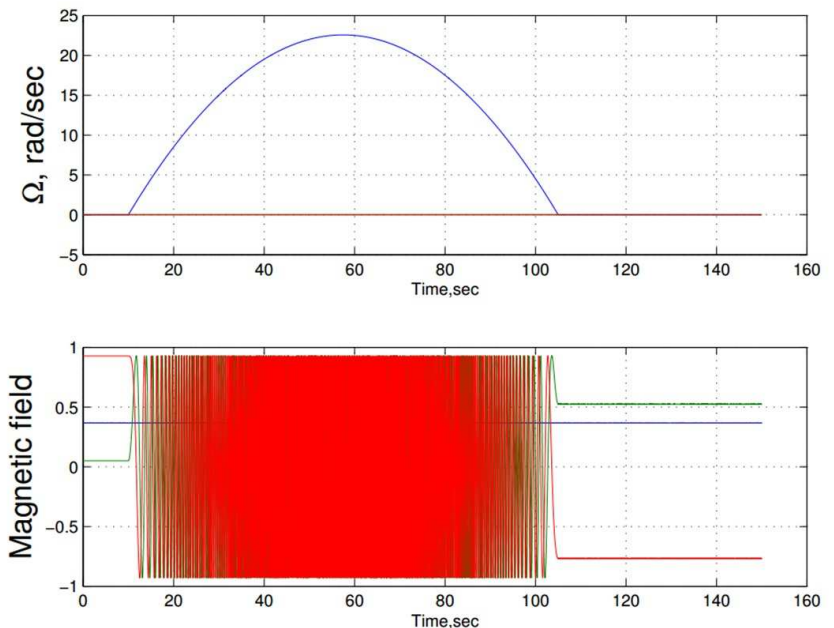

Fig. 2. Graphs of sensor signals during the simulation

The graphs in Figure 3 show the simulation results. The upper graph shows the pitch angle and angles calculated from the magnetometer and the gyroscope readings with the Kalman correction. The second graph shows the error in the roll angle in radians. The third and fourth graphs represent the estimates of the zero shift and gyroscope scale factor, respectively. From the graphs analysis, it can be concluded that the algorithm is able to effectively evaluate and compensate the coefficients of the gyro error model.
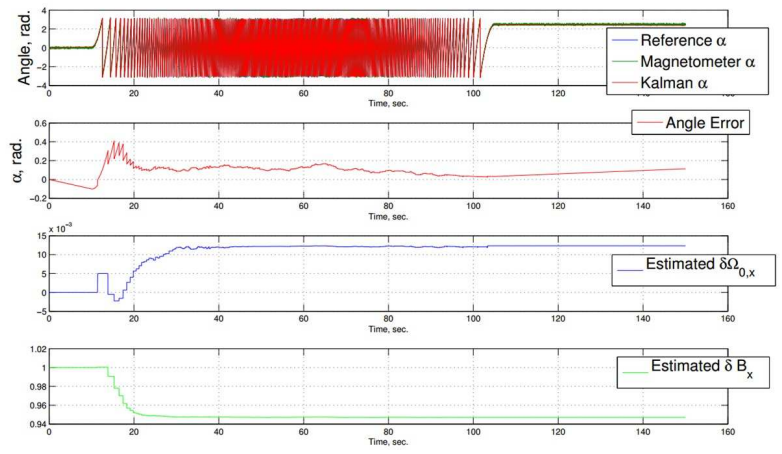

Fig. 3. Simulation results of the magnetometer correction algorithm

\section{Modeling algorithm with optical sensor correction}

The simulation conditions in this case are similar to the conditions for modeling the algorithm with the magnetometer correction. Figure 4 shows the simulation results for the optical sensor correction algorithm.

From the graphs analysis, it can be seen that in spite of a much rarer correction (once for a complete revolution), in this case it is possible to estimate and to cancel the AVS errors.
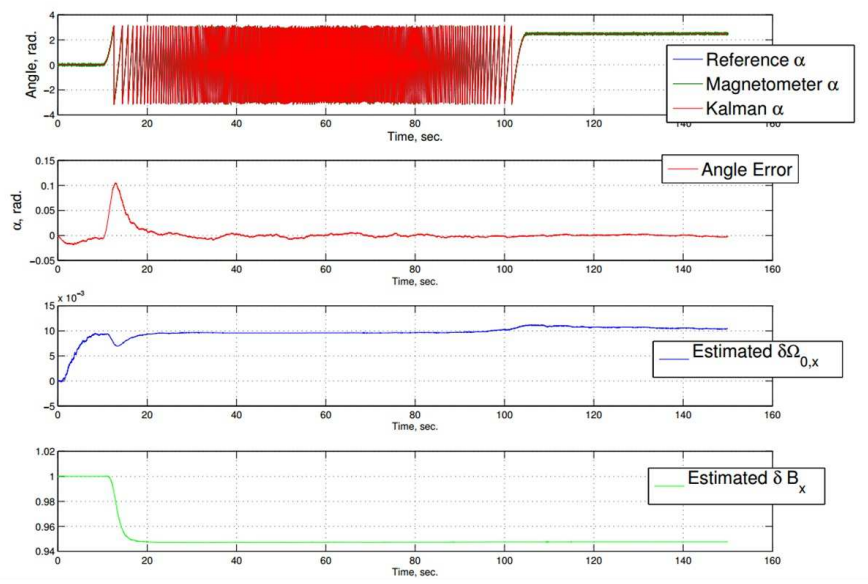

Fig. 4. Simulation results of the optical sensor correction algorithm

\section{Experimental research facility}

Laboratory motion simulator was created specifically for experiments (Figure 5). The motor provides rotation of the measurement unit at a high angular velocity (7,000-10,000 degrees per second). The measurement unit consists of a main gyroscope located in a special position - to measure rotation around the main axis. Also, the measurement unit includes two accelerometers arranged mutually orthogonally, in the plane which perpendicular to the axis of rotation. Two magnetometric sensors are located in a similar way, and two light sensors are also present in the unit. All information is collected by the controller and written to flash memory. The unit has autonomous power from the batteries and there is no need to broadcast data from the sensors through the rotary mechanisms at such high speed. Otherwise, the information would certainly be distorted. Inside the sensor unit, there is also a reference optical encoder. It registers the rotation of the block relative to the fixed limb with 24 slits per full revolution.

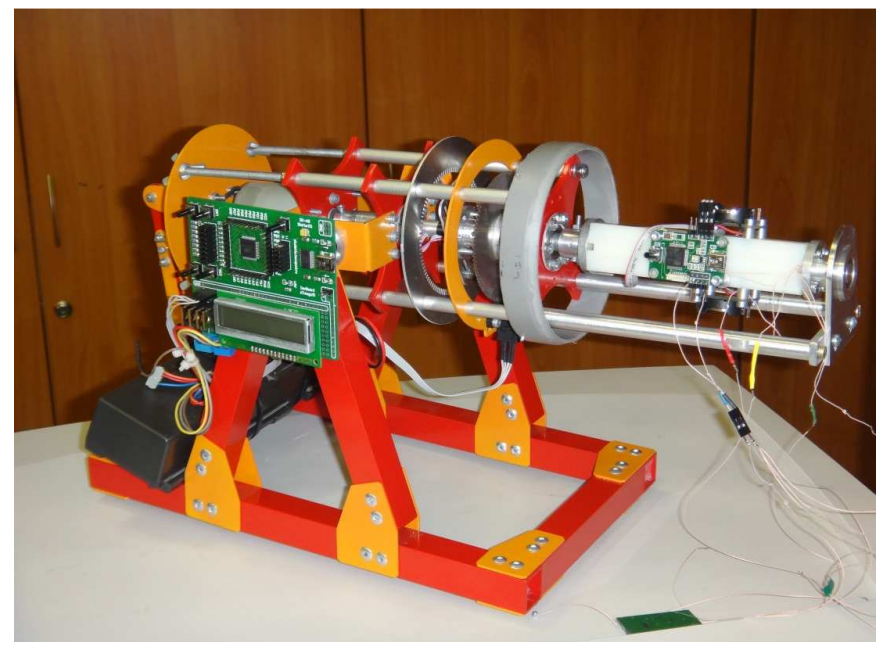

Fig. 5. Special motion simulator 
Thus, not only the primary inertial data are recorded on the internal flash memory of the experimental block, but also the reference data on the actual angular position. All data with minimal time delays are written synchronously to one memory device along with timestamps. After an experiment or a series of experiments, it becomes possible to repeatedly develop the algorithm for determining the roll and comparing it with the actual results. The discreteness of the reference position sensor is 15 degrees.

\section{Experimental results}

After the first experiments, the problem of automatic calibration of magnetometric sensors was sharply raised, it is not possible to trust magnetometers in all conditions, and it is not always possible to correctly calibrate them, even if they perform a complete rotation. The elements of the test bench significantly distort the Earth's magnetic field, and the engine even creates its own, nevertheless some success in correction by magnetometric sensors was achieved. Figure 6 shows a two-dimensional calibration of magnetometers (the Earth's magnetic field with respect to two sensor axes). In a homogeneous magnetic field, the pattern resembles a circle, after calibration, the circle has a radius close to unity and smoother contours.

The experiment showed that in a fairly homogeneous magnetic field, it is possible to make adequate estimates of the errors of the zero signal and the scale factor of the micromechanical gyro using a magnetometer. Probably a similar conclusion can be made with the use of optical sensors. Figure 7 shows the resulting experimental data with measured angles.

\section{III.DISCUSSION}

According to the simulation and experiment, it is assumed that in a number of conditions it is rational to estimate the correction of the micromechanical gyro, based on the data of magnetometric sensors. To estimate the error of the scale factor and the zero signal of the gyro, it is also possible to use a system based on optical sensors and an accelerometer. Probably, the best quality of the entire system can be achieved with a rational combination of different types of correction and automatic selection of the most accurate based on current conditions. To assess the adequacy of the source of correction, it is necessary to formulate eligibility criteria (adequacy of the corrector). Such criterion for magnetometers can be a twodimensional calibration picture. If it is fundamentally different from the circle, then the use of these sensors is not desirable.

It is proposed to use a system, based on the gyro and magnetometer or accelerometer, to compensate the error in the scale factor and zero signal. In this case, the accelerometer will serve as a source of corrective information about object rotation speed around the longitudinal axis. Gyro must be installed along the longitudinal rotation axis, and the accelerometer - perpendicular to the longitudinal rotation axis $[6,10]$.
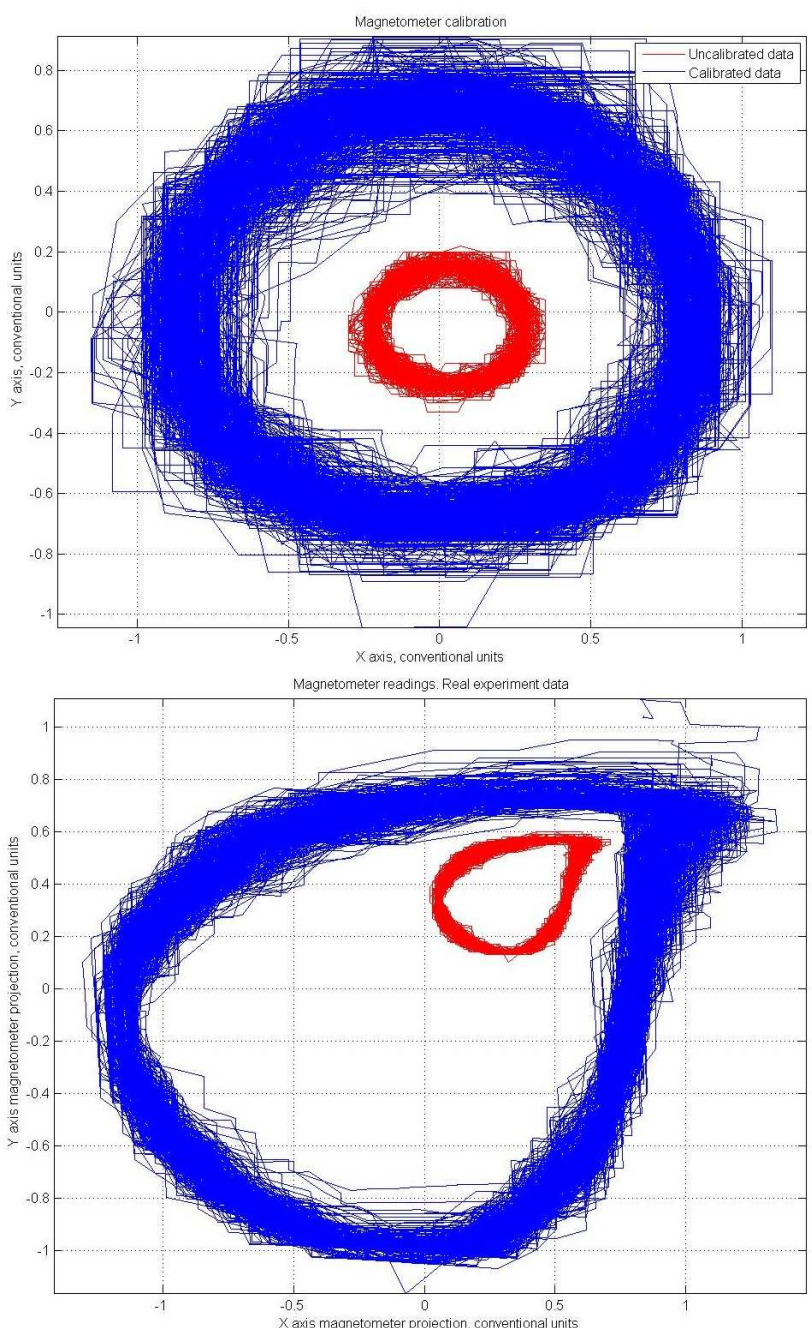

Fig. 6. Automatic calibration of the magnetometric sensor. From above - in a homogeneous normal magnetic field, on the right - in a distorted magnetic field.

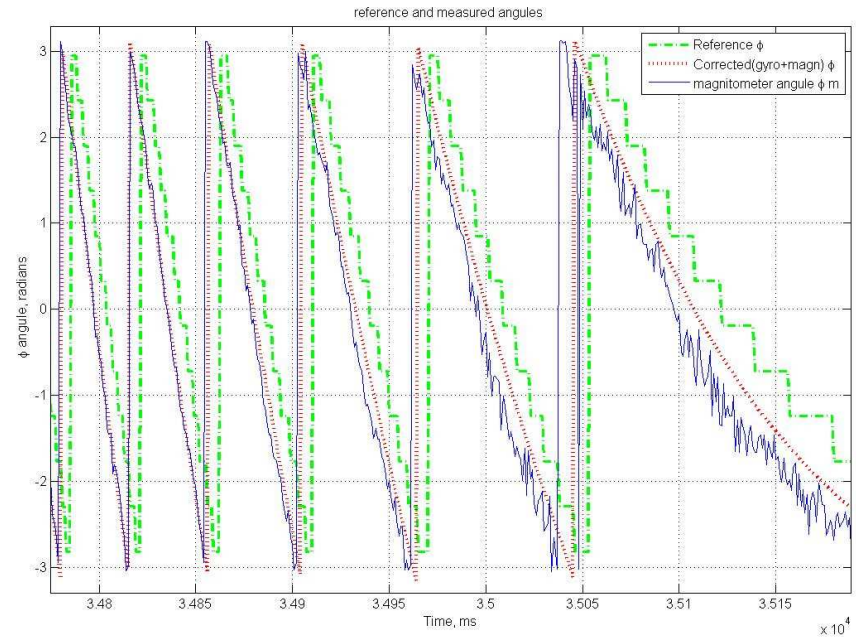

Fig. 7. Measurements of the angle of roll with a magnetometer and a complex sensor (MEMS DUS + magnetometer) in comparison with the reference encoder. 


\section{IV.CONCLUSION}

Successful simulation for the operation of a single-angle orientation system for determining the roll angle of a fastrotating object has been carried out. The results, taking into account the actual levels of modern sensors errors, show that the problem can be solved by correcting the AVS signal with magnetometer data or optical sensor data. It is supposed to continue system modulation with accelerometers correction and work on creating a layout of the orientation system.

\section{ACKNOWLEDGMENT}

This work was financially supported by the Russian Science Foundation. A unique identifier for applied research is 16-19-00177.

\section{REFERENCES}

[1] Y.A. Chaplygin, Nanotechnology in Electronics, Issue 3, Moscow: Technosphere, 2015, 480 p.

[2] V.K. Varadan, K.J.Vinoy, K.A. Jose, RF MEMS and Their Applications. West Sussex: John Wiley\& Sons Ltd, 2003, 528 p.
[3] Y.A. Chaplygin, Nanotechnology in Electronics. Issue 2, Moscow: Technosphere, 2013, $688 \mathrm{p}$.

[4] K.K. Veremeenko, I.A. Galay, "Development of an algorithm for calibrating an inertial navigation system on a biaxial test bed", Electronic Journal "MAI Proceedings", Issue number 63, http://trudymai.ru/published.php?ID=36139

[5] NASA Space Vehicle Design Criteria (Guidance and Control), "Spacecraft Earth Horizon Sensors," NASASP-8033, NASA, pp.42-46, December 1969.

[6] J. Rogers, M. Costello, and D. Hepner, "A roll orientation estimator for smart projectiles using thermopile sensors," Journal of Guidance, Control, and Dynamics, vol. 34, pp. 688-697, May-June 2011.

[7] K.K.Veremeenko, S.Yu. Zheltov, N.V. Kim, G.G. Sebryakov, M.N. Krasil'shchikov, Modern Information Technologies in the Tasks of Navigation and Guidance of Unmanned Maneuverable aircraft, Moscow: Fizmatlit, 2009, 556 p.

[8] Control and Guidance of Unmanned Maneuverable Aircraft Based on Modern Information Technologies, Ed. Krasil'shchikov M.N., Sebryakov G.G., Moscow: Fizmatlit, 2003, 280 p.

[9] E. Belousov, Y. Krouglov, A. Timoshenkov, "Current Driver for MEMS Angular Sensor", 2015 Internet Technologies and Applications (ITA) Proceedings [Sixth International Conference (ITA 15), pp. 349-351].

[10] A. Shalimov, S.Timoshenkov, N. Korobova, M. Golovinskiy, A. Timoshenkov, E. Zuev, S. Berezueva, A. Kosolapov, "Comb structure analysis of the capacitive sensitive element in MEMS - accelerometer", Proceedings [SPIE. Int, Soc. for Opt.Eng. January 2015, 9467, 94672W]. 